\title{
Author Correction: Priority list of biodiversity metrics to observe from space
}

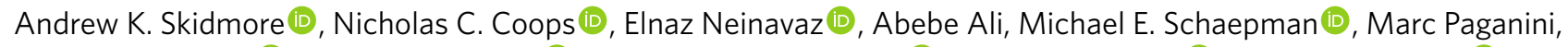

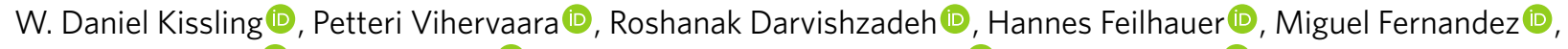
Néstor Fernández (1), Noel Gorelick (1D), Ilse Geijzendorffer, Uta Heiden (1), Marco Heurich (1), Donald Hobern, Stefanie Holzwarth (1), Frank E. Muller-Karger (D), Ruben Van De Kerchove, Angela Lausch (1), Pedro J. Leitão (D, Marcelle C. Lock (1D, Caspar A. Mücher, Brian O'Connor, Duccio Rocchini (i), Woody Turner, Jan Kees Vis, Tiejun Wang (i), Martin Wegmann (1D and Vladimir Wingate

Correction to: Nature Ecology \& Evolution https://doi.org/10.1038/s41559-021-01451-x, published online 13 May 2021.

In the version of this Perspective originally published, the names of authors Pedro J. Leitão and Ilse Geijzendorffer were mistakenly written as Pedro J. Leitãu and Ilse Geizendorffer, respectively. These have now been corrected.

Published online: 24 May 2021

https://doi.org/10.1038/s41559-021-01492-2

(c) Springer Nature Limited 2021 